\title{
Optimal Control of Engine Warmup in Hybrid Vehicles
}

\author{
Vital van Reeven ${ }^{1,2 *}$, Theo Hofman ${ }^{1}$, Frank Willems ${ }^{1}$, Rudolf Huisman ${ }^{2}$ and Maarten Steinbuch ${ }^{1}$ \\ ${ }^{1}$ Control Systems Technology, Eindhoven University of Technology, P.O. Box 513, 5600 MB Eindhoven - The Netherlands \\ ${ }^{2}$ DAF Trucks N.V., Eindhoven - The Netherlands \\ e-mail:v.v.reeven@tue.nl - t.hofman@tue.nl - f.p.t.willems@tue.nl - rudolf.huisman@daftrucks.com - m.steinbuch@tue.nl \\ * Corresponding author
}

\begin{abstract}
An Internal Combustion Engine (ICE) under cold conditions experiences increased friction losses due to a high viscosity of the lubricant. With the additional control freedom present in hybrid electric vehicles, the losses during warmup can be minimized and fuel can be saved.

In this paper, firstly, a control-oriented model of the ICE, describing the warmup behavior, is developed and validated on measured vehicle data. Secondly, the two-state, non-autonomous fuel optimization, for a parallel hybrid electric vehicle with stop-start functionality, is solved using optimal control theory. The principal behavior of the Lagrange multipliers is explicitly derived, including the discontinuities (jumps) that are caused by the constraints on the lubricant temperature and the energy in the battery system. The minimization of the Hamiltonian for this two-state problem is also explicitly solved, resulting in a computationally efficient algorithm. The optimal controller shows the fuel benefit, as a function of the initial temperature, for a long-haul truck simulated on the FTP-75.
\end{abstract}

Résumé - Contrôle optimal d'échauffement du moteur dans les véhicules hybrides — Un moteur à combustion interne (ICE, Internal Combustion Engine) en condition froide provoque une croissance de pertes de frottement suite à la viscosité élevée du lubrifiant. Dues à la commande libre supplémentaire appliquée dans les véhicules électriques hybrides, les pertes d'échauffement peuvent être réduites au maximum tout en économisant du carburant.

Dans cet article, premièrement, un modèle de contrôle de l'ICE, décrivant le comportement d'échauffement, a été développé et validé par les données mesurées sur le moteur. Deuxièmement, dans un véhicule électrique hybride parallèle avec la fonctionnalité d'arrêt-démarrage, l'optimisation du double-état, non-autonome, du carburant est résolue en appliquant la théorie du contrôle optimal. Le comportement principal des multiplicateurs de Lagrange est explicitement dérivé, y compris les discontinuités (sauts) qui sont causées par les contraintes de la température du lubrifiant et par la capacité dans le système de batterie. La minimisation de l'Hamiltonien de ce problème de doubleétat est aussi explicitement résolue, résultant en un algorithme de calcul efficace. Le régulateur optimal indique l'économie du carburant en fonction de la température initiale, pour un grand routier simulé sur le FTP-75. 


\section{INTRODUCTION}

Hybrid Electric Vehicles (HEV) have emerged as a promising solution to reduce operational cost in commercial road transportation, while complying to increasingly stringent emission legislation. Since HEV have more than one power converter, they offer additional control freedom, compared to conventional vehicles, which gives opportunities for the Energy Management System (EMS) to decrease fuel consumption and emissions. The EMS has to consider the energy relevant systems of the hybrid system and already a large amount of solutions have been proposed that take the battery energy dynamics into account [1]. However, the system efficiency can be further improved by taking additional systems into account as suggested by [2] and [3], referred to as a 'unified', 'integrated', 'total' or 'holistic' energy management. Examples of such additional systems are the battery with its temperature and aging characteristics, engine aftertreatment system, waste-heat recovery system, combustion engine or the cabin heater [4-7].

Including model information of these systems in the controller, increases the complexity of the optimization to perform, while the computational burden must be limited for real-time control. However by using optimal control techniques based on the Pontryagin Minimum Principle (PMP) $[8,9]$, the global optimization is reduced to a local optimization, accompanied by finding one or more Lagrange multipliers or co-states. For the one state problem [10], shows that the local optimization can be made explicit, whereas [11] and [12] show that the Lagrange multiplier of the battery has very simple dynamics, i.e. piecewise constant for a constrained energy buffer. They show that a close-to-optimal solution can be efficiently calculated, when some simplifications to the control models are made.

Extending these methods to more states is not trivial and the behavior of multiple Lagrange multipliers is subject of several publications. On the one hand, heuristics or classical controllers (PI) are used to determine the multipliers, which are inherently sub-optimal, but readily real-time implementable $[6,13]$. On the other hand, in [7, 14], explicit formulations of the multipliers are used to show the principle behavior of the optimal solution, and the remaining twopoint boundary value problem is solved for a predetermined drive cycle.

At low temperatures the efficiency of the ICE is reduced, due to increased friction losses, and during warmup of the ICE in a HEV, a trade-off exists between fast warmup and efficient fuel use. In this paper, a fuel optimal EMS is developed, which takes the battery energy and the temperature state of the ICE into account. The work of [7] is thereby extended to include state constraints and additional necessary conditions are formulated. The warmup behavior of the ICE is described and validated with a low-complexity, parametric control model, which enables the explicit formulation of the Lagrange multiplier. Furthermore, the local optimization is solved explicitly, thereby extending the work of [10] with an additional state.

In Section 1, we derive and validate the control model for ICE warmup behavior, and describe the components in the hybrid driveline. Section 2 defines six scenarios to illustrate the fuel saving mechanism, under varying driving conditions and EMS solutions. In Section 3, the state constrained optimal control problem is explicitly solved. Simulation results in Section 4 show the fuel benefit under typical driving conditions, where the last section discusses the results of this work.

\section{CONTROL-ORIENTED HYBRID VEHICLE MODEL}

The efficiencies of the power converters (ICE and MG) have a large influence on the optimal decision of the EMS. For the ICE, it is known that the temperature $T$ of the lubricant has a large influence on the friction, and consequently the mechanical efficiency. In this work, the influence of adding the temperature $T$ to the optimal control problem is investigated. Therefore, a low complexity, control oriented model of the ICE is developed and validated with measurements. The Motor/Generator and battery models complete the HEV model.

The parallel hybrid topology is schematically depicted in Figure 1. The fuel power $P_{f}$ flows from the tank $(\mathrm{F})$ to the ICE, converting it to mechanical power $P_{e}$. Through the clutch (CL), the power from the Motor/Generator (MG) $P_{m}$ is added, resulting in the power at the power split point $P_{p}$. This power is transferred through the Gearbox (GB), final drive and wheels to the road (R): the demanded power $P_{d}$. The MG exchanges electrical power $P_{b}$ with the battery (B). Two states of the system, $E_{T}$ (which is proportional to the temperature of the ICE) and $E_{b}$ (which is the energy in the battery), are explicitly incorporated in the EMS, while

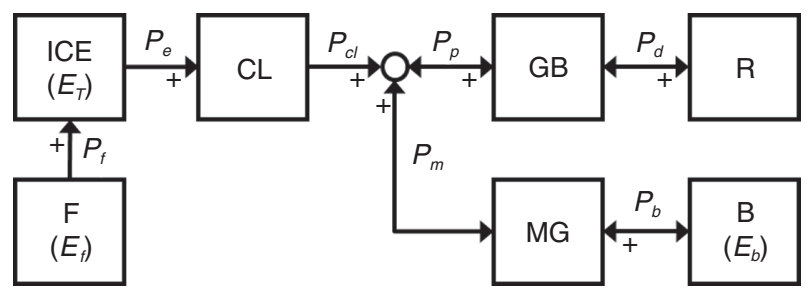

Figure 1

Topology of the parallel HEV with its relevant power flows, and the considered states (in brackets). 
TABLE 1

Vehicle parameters for simulation

\begin{tabular}{c|c|c|c}
\hline & Value & Unit & Description \\
\hline$\rho$ & $2.5 \mathrm{e} 4$ & $\mathrm{~kg}$ & Mass of vehicle \\
\hline$c_{w} A$ & 1.2 & $\mathrm{~kg} / \mathrm{m}^{3}$ & Air density \\
\hline$c_{r}$ & $4 \mathrm{e}-3$ & - & Air drag coefficient times frontal area \\
\hline$\overline{P_{d}}$ & $3 \mathrm{e} 5$ & $\mathrm{~W}$ & Rolling resistance coefficient \\
\hline$\frac{P_{d}}{G}$ & $-5 \mathrm{e} 4$ & $\mathrm{~W}$ & Maximum power of drivetrain \\
\hline$\overline{E_{b}}-\underline{E_{b}}$ & 4 & $\mathrm{MJ}$ & Minimum power of drivetrain \\
\hline
\end{tabular}

minimizing the fuel energy $E_{f}$ over a drive cycle. The HEV under consideration is a heavy duty commercial vehicle, with typical vehicle parameters denoted in Table 1.

\subsection{Internal Combustion Engine during Warm-Up}

When the ICE is started after a long time of inactivity, the temperature of the ICE will be close to ambient temperature. At these cold conditions, the lubricant in the engine is highly viscous, resulting in increased friction losses. The ideal working temperature of the ICE is however much higher, typically $90^{\circ} \mathrm{C}$. To describe the warm-up effects, a model is derived for the relevant thermal dynamics of the ICE and the influence on the efficiency.

The efficiency of the ICE is often separated in a thermodynamic term and a friction term [7, 14-16]. For a petrol engine the thermodynamic efficiency drops at low temperatures due to wall wetting, an effect that does not occur in Diesel engines. Consequently, low temperatures in Diesel engines mainly influence the friction [15], whereas in $[14,17]$ for a petrol engine both thermodynamic efficiency and friction are temperature dependent. In [18], the viscosity of the engine lubricant is identified as the largest contributor of increased friction at cold and, like [19], he extends friction modeling of the warm ICE [20], to cold conditions. It should be noted that due to the complexity of the physical mechanisms, the models are of an empirical nature. These models have however in common that the relation between temperature and viscosity is tabulated, which is followed by [7, 14]. In [15], a parametric dependency is noted, but not specified. However, in [21], an overview is given of parametric models describing the temperature-viscosity relation, but not applied to engine friction models.

In this section, a parametric, control-oriented model of the ICE is proposed, that describes the warmup effect with a low

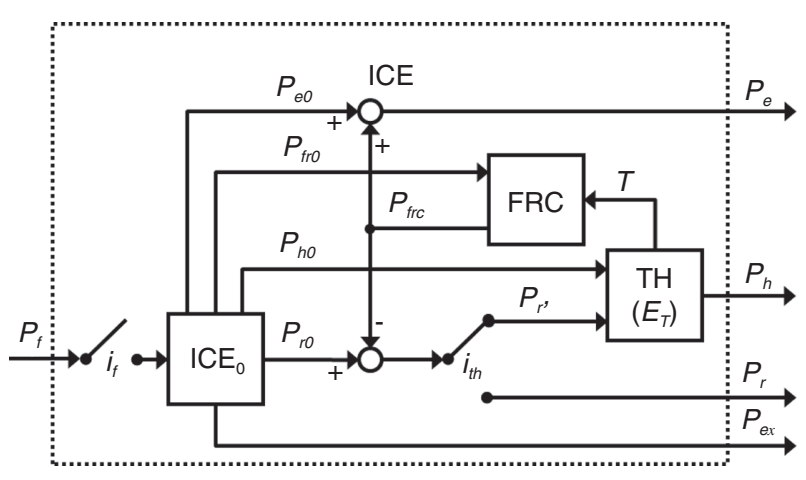

Figure 2

Schematic of the thermal ICE model, in which the nominal model $\mathrm{ICE}_{0}$ of the warm engine, is corrected for friction due to cold (FRC), based on the lumped temperature model $\mathrm{TH}$.

amount of parameters. A schematic power flow scheme of the ICE model is given in Figure 2. At the top-level, i.e. outside the dotted box, fuel power $\left(P_{f}\right)$ is converted to mechanical work $\left(P_{e}\right)$, heat losses to the surrounding, comprising convection, radiation and conduction $\left(P_{h}\right)$, cooling to the radiator $\left(P_{r}\right)$ and heat flow to the exhaust and intercooler $\left(P_{e x}\right)$, which closes the stationary energy balance $P_{f}=P_{e}+P_{h}+P_{r}+P_{e x}$.

Inside the dotted box, the nominal model $\mathrm{ICE}_{0}$ is corrected for non-nominal, cold conditions, i.e. with temperatures below the nominal value. The parameters for the nominal condition are denoted with the subscript 0 . The temperatures of the lubricant, coolant and engine block are lumped in one temperature state $E_{T}$. The correction consists of a friction term due to cold (FRC), that acts both on the mechanical output $P_{e 0}$, as on the heat release into the coolant $P_{r 0}$. In the next sub-sections, the nominal model $\mathrm{ICE}_{0}$, the friction-due-to-cold model FRC and the thermal model TH are described.

\subsection{Nominal Model (ICE)}

The nominal $\mathrm{ICE}_{0}$ model describes the fuel power $P_{f}$ needed to deliver mechanical power $P_{e 0}$, dependent on engine speed $(\omega)$ and torque $(\tau)$ for a warm engine. For Diesel engines a Willans approximation describes the first order behavior of the engine very well [15]. This approximation separates the thermodynamic losses from the mechanical losses (friction, gas exchange). The thermodynamic efficiency (or indicated efficiency [16]) $\eta$, is close to constant for varying $\tau$, as Diesel engines do not apply fuel enrichment at full load. 


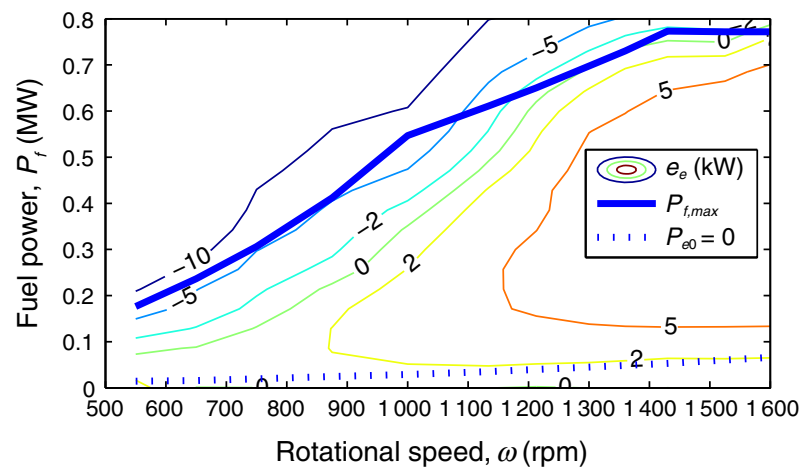

Figure 3

Nominal ICE model error $e_{e}$ in $\mathrm{kW}$, with maximum fueling $P_{f, \max }$ and fueling at no-load $\left(P_{e 0}=0\right)$.

Assuming small engine speed variations $\omega, \eta$ is constant, leading to:

$$
P_{f}(\omega, \tau)=\frac{1}{\eta}\left(P_{e 0}(\omega, \tau)-P_{f r 0}(\omega)\right)
$$

where $P_{f r 0}$ is the nominal friction, which is a (mapped) function of $\omega$ and, by definition, $P_{f r 0} \leq 0$.

Stop-start functionality of the HEV is controlled with switch $i_{f}$. If fuel is cut off $\left(i_{f}=0\right)$, we assume that the ICE is instantaneously disconnected from the driveline with CL and stopped: $\omega=0$. Consequently $P_{f r 0}(0)=0$ and the nominal ICE model can be written as:

$$
P_{f}\left(i_{f}, \omega, \tau\right)=\frac{i_{f}}{\eta}\left(P_{e 0}(\omega, \tau)-P_{f r 0}(\omega)\right)
$$

The model is fitted to stationary test bench measurements, where the load $P_{e}$ is varied over the operating range. In Figure 3, the error $e_{e}$ between model and measurement:

$$
e_{e}(\omega, \tau)=P_{e 0, \text { meas }}(\omega, \tau)-P_{e 0}(\omega, \tau)
$$

is shown, where subscript 'meas' denotes the measured values. For a large operating range, $\left|e_{e}\right|$ is below $5 \mathrm{~kW}$, which is below $1.7 \%$ for a $300 \mathrm{~kW}$ engine. The maximum load on the engine is limited in fuel, denoted by $P_{f, \max }$. As in our model both $\eta$ as $P_{f r 0}$ are not depending on $\tau,-P_{f r 0}$ is equivalent to $P_{e 0}=0$.

\subsection{Friction Model (FRC)}

Friction models from e.g. [20] consider the engine at a thermal equilibrium $T=T_{0}$. At cold conditions, the viscosity of the lubricant is increased, causing an increase in friction.
In $[18,19]$, a temperature dependent correction is proposed to the nominal viscosity $\mu\left(T_{0}\right)$ :

$$
V_{c}=\left(\frac{\mu(T)}{\mu\left(T_{0}\right)}\right)^{x}
$$

with $V_{c}$ the correction term, $\mu\left(T_{0}\right)$ the viscosity of warm lubricant, $\mu(T)$ the viscosity as function of temperature and $x$ an exponent ranging from 0.3 to $0.5[18,19]$. Several relations between temperature and viscosity are given in [21], where we select:

$$
\mu(T)=R e^{-\alpha^{\prime} T}
$$

with $\alpha^{\prime}$ an empirical constant. Combining (4) and (5), results in the temperature dependent friction correction:

$$
V_{c}(T)=e^{-\alpha\left(T-T_{0}\right)}
$$

with $\alpha$ an empirical constant. It should be noted that $V_{c}$ is unity for a warm engine $\left(T=T_{0}\right)$. Furthermore the friction could be reduced by increasing the temperature above $T_{0}$, however this causes oil degradation and should be avoided.

Not all friction in the engine consists of viscous friction, as shown by [20], and accordingly not all friction must be corrected (opposed to [14]). Therefore we propose the parameter $\gamma$, that determines the fraction of the total friction influenced by temperature:

$$
P_{f r}(\omega, T)=\left(\gamma V_{c}(T)+(1-\gamma)\right) \cdot P_{f r 0}\left(\omega, T_{0}\right)
$$

Now we define the friction due to cold $P_{\text {frc }}$ as:

$$
P_{f r c}(\omega, T)=P_{f r}(\omega, T)-P_{f r 0}\left(\omega, T_{0}\right)
$$

which is the amount of friction caused by temperatures below $T_{0}$, and with (7) results in:

$$
P_{f r c}(\omega, T)=\gamma\left(V_{c}(T)-1\right) \cdot P_{f r 0}\left(\omega, T_{0}\right)
$$

The total friction losses due to cold are defined as:

$$
E_{f r c}(\omega, T, t)=\int_{t_{0}}^{t_{1}\left(T_{0}\right)} P_{f r c}(\omega, T) d t
$$

which represents the energy that is lost in additional friction losses during the warmup period of the engine $\left[t_{0}, t_{1}\right]$, where $t_{1}$ is the first moment that $T_{0}$ is reached. Note that after $t_{1}$, $T-T_{0}=0, V_{c}=1, P_{f r c}=0$ and $E_{f r c}$ will remain constant.

Combining (2) with $P_{e}=P_{e 0}+P_{f r c}$ results in:

$P_{f}\left(i_{f}, \omega, \tau, T\right)=\frac{i_{f}}{\eta}\left(P_{e}(\omega, \tau)-P_{f r 0}(\omega)-P_{f r c}(\omega, T)\right)$

with $P_{f r c}$ as in (9). 


\subsection{Thermal Model (TH)}

The thermal friction model uses the temperature of the lubricant. During warmup of the engine the heat exchange between lubricant, coolant and engine block is fast, causing the temperatures to follow each other closely. Therefore we model one lumped thermal mass, and use the lumped temperature for the friction model.

The model includes a switching thermostat $i_{t h}$, Figure 2 . During nominal conditions, the thermostat is active $\left(i_{t h}=1\right)$ and is perfectly controlling the temperature $\left(T=T_{0}\right)$ :

$$
\left.\begin{array}{c}
P_{f r c}=0 \\
P_{r^{\prime}}=0 \\
P_{r}=P_{r 0} \\
P_{h}=P_{h 0}=\theta\left(T_{0}-T_{a m b}\right)
\end{array}\right\} \text { if } i_{t h}=1
$$

where $P_{h 0}$ is the heat removed from the engine to keep $T_{0}$ constant, $\theta$ the heat transfer coefficient to the surroundings and $T_{a m b}$ the ambient temperature. The heat released to the coolant under nominal conditions is approximated by:

$$
P_{r 0}=\kappa P_{f}
$$

where $\kappa$ is a constant ratio. During cold conditions $\left(T<T_{0}\right)$, the thermostat is not active $\left(i_{t h}=0\right)$ and no heat is removed by the radiator $\left(P_{r}=0\right)$ :

$$
\left.\begin{array}{c}
P_{r}=0 \\
P_{r^{\prime}}=P_{r 0}-P_{f r c} \\
P_{h}=\theta\left(T-T_{a m b}\right) \\
P_{h 0}=\theta\left(T_{0}-T_{a m b}\right)
\end{array}\right\} \text { if } i_{t h}=0
$$

The change in thermal energy of the engine is defined as:

$$
\dot{E}_{T}=C \dot{T}=-P_{T}
$$

where $C$ is the heat capacity and:

$$
P_{T}=P_{h}-P_{h 0}-P_{r^{\prime}}
$$

Note that $P_{T}>0$ discharges the thermal buffer $E_{T}$. Combining $(12-14,16)$ results in:

$$
P_{T}=\left\{\begin{array}{cc}
P_{h}-P_{h 0}-\kappa P_{f}+P_{f r c} & \text { if } i_{t h}=0 \\
P_{h}-P_{h 0} & \text { if } i_{t h}=1
\end{array}\right.
$$

\subsection{Validation of Thermal Behavior}

The thermal ICE model parameters $\kappa, C, \gamma, \alpha, \theta$ are fitted on measurements, performed on a test bench. To validate the

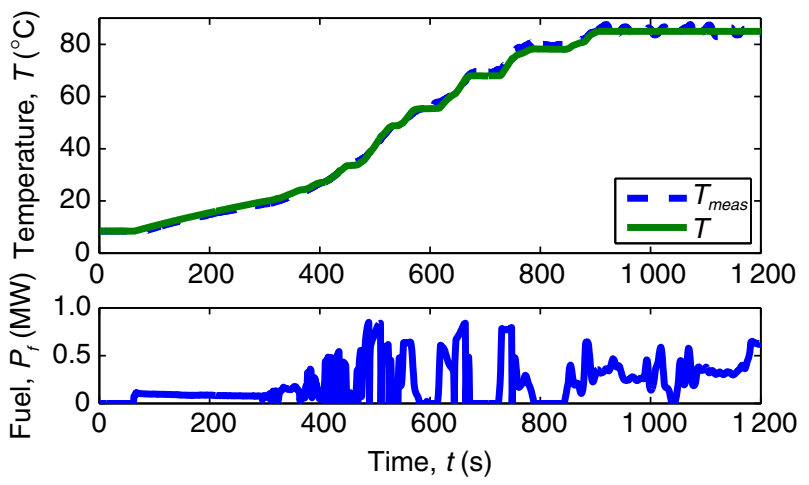

Figure 4

ICE thermal model validation on a long-haul vehicle.

non-nominal ICE model, several long-haul vehicles are monitored throughout the year, leading to a data set with $T$ ranging $[0,90]^{\circ} \mathrm{C}$. From this data set, warmup events are selected, where $T$ has a range of at least $[30,80]^{\circ} \mathrm{C}$. For one typical warmup event, the time traces are shown in Figure 4. During this typical long-haul event the vehicle is idling up to $t=300 \mathrm{~s}$, then driving through the city and continues at $t=850 \mathrm{~s}$ on the highway. The modeled temperature as a function of $P_{f}, \omega, T_{a m b}$, follows closely the measured engine temperature.

For a total of 169 warmup events, the average error between measured and modeled $T$ :

$$
e_{T}=\frac{1}{N} \sum_{i=1}^{N}\left(T_{\text {meas }}(i)-T(i)\right)
$$

with $N$ the number of measurements up to $t_{1}$, is calculated. The histogram of ' $e_{T}$ with $P_{h}$ ' is shown in Figure 5. We can observe that for a large number of events, $e_{T}$ is reasonably small: $50 \%$ falls within $[-2,3]^{\circ} \mathrm{C}$. Some events however have considerably larger error for two reasons. Firstly, as the model has integrating behavior, small biases in the model, result in large errors over time. Secondly, the model ignores the air speed around the ICE, which is known to influence $P_{h}$ [22]. With the vehicle speed as a measure for the air speed, the solid line in Figure 5 shows a clear correlation between the model error and the vehicle speed. It also shows that the conditions for the test bench measurements $\left(e_{T}=0\right)$, used for fitting the parameters, are equivalent to an average vehicle speed of $12 \mathrm{~m} / \mathrm{s}$.

Instead of elaborating the plant model with speed dependent convection, $P_{h}$ is set to zero, i.e. we assume a perfectly isolated engine. The reason is, that $P_{h}$ is very small during average operation of the ICE. Only when the ICE is stopped for a prolonged time, e.g. parking overnight, $P_{h}$ will cause a 


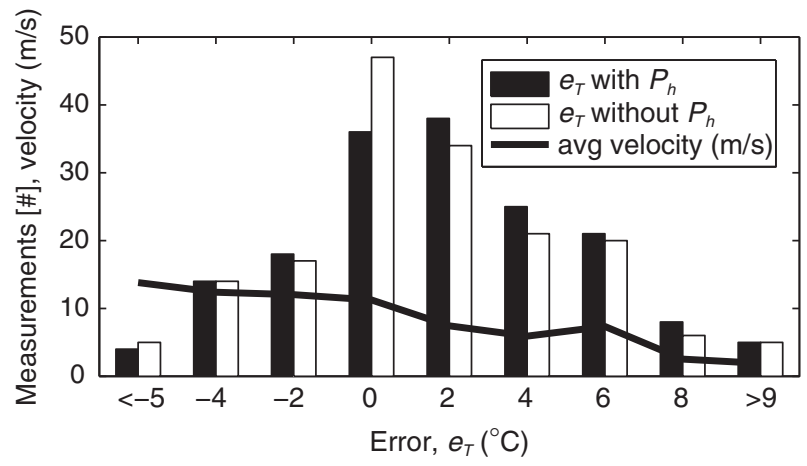

Figure 5

ICE thermal model validation 'with' and 'without' $P_{h}$, on an annual data set of several long-haul vehicles.

significant drop in $T$. Stop-start events in a hybrid are typically short, thus making the contribution of $P_{h}$ small in the energy balance.

As a consequence of $P_{h}=0$, the control model is not able to cool down: when the engine is stopped, $P_{f}$ and $P_{f r 0}$ are zero, and the temperature remains constant. The validation error histogram for the model ' $e_{T}$ without $P_{h}$ ', as shown in Figure 5, is smaller than for the model including $P_{h}$, which justifies the simplification. Furthermore, when the vehicle speed does not change between comparisons, as in Section 4, no vehicle speed dependent errors influence the EMS performance.

\subsection{Battery, Motor Generator and Gearbox Model}

The battery is modeled as an integrator, with quadratic losses, [10]:

$$
\begin{gathered}
\frac{d E_{b}}{d t}=-P_{b i} \\
P_{b i}=P_{b}+\beta P_{b}^{2}
\end{gathered}
$$

with $E_{b}$ the energy in the battery, $P_{b i}$ the internal battery power, $P_{b}$ the power at the terminals and $\beta$ the loss constant. The loss constant is fitted to a high fidelity model of the Li-ion battery manufacturer, with the averaged model error:

$$
e_{b}=P_{b, \text { meas }}-P_{b}
$$

shown in Figure 6. The model has an error in the same order of magnitude as the ICE model (typically below $2 \mathrm{~kW}$ ), and

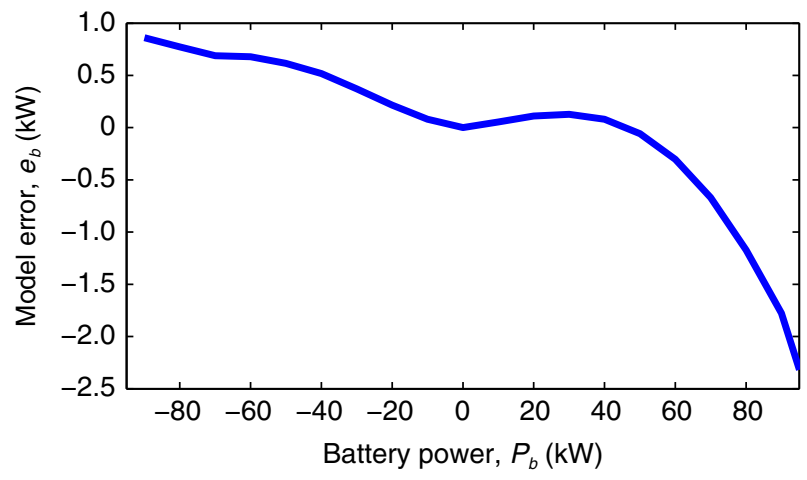

Figure 6

Battery model error $e_{b}$ in $\mathrm{kW}$.

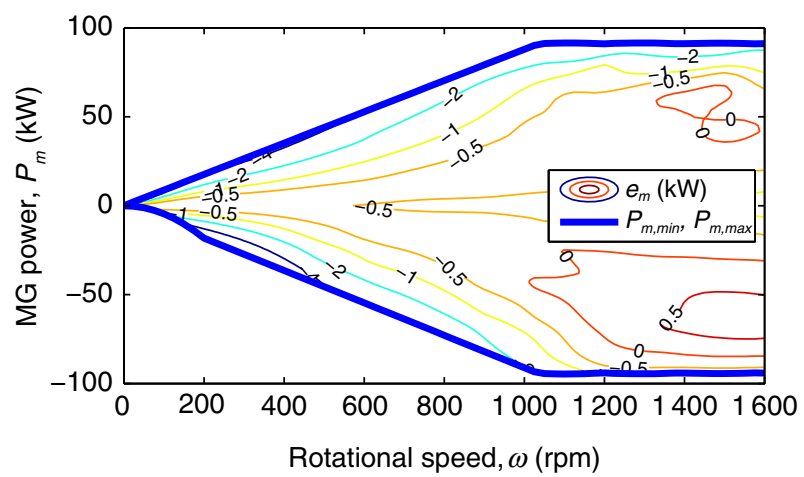

Figure 7

MG model error $e_{m}$ in $\mathrm{kW}$.

has thus comparable fidelity. Note that $P_{b}>0$ discharges the battery.

The mechanical output of the MG is modeled as:

$$
P_{m}= \begin{cases}\eta_{m} P_{b}, & \text { if } P_{b}>0 \\ \frac{1}{\eta_{m}} P_{b}, & \text { if } P_{b} \leq 0\end{cases}
$$

with $P_{m}$ the MG power connecting to the powersplit device, $P_{b}$ the electrical power from the battery and $\eta_{m}$ the (constant) efficiency of the MG. The mechanical power limits $P_{m, \min }, P_{m, \max }$ are mapped as a function of $\omega$. The error between the model and measurements:

$$
e_{m}=P_{m, \text { meas }}-P_{m}
$$

is shown in Figure 7. The model has an error in the same order of magnitude as the ICE and battery model 
(typically below $2 \mathrm{~kW}$ ), and has thus comparable fidelity.

The gearbox is modeled as a continuous variable ratio with $100 \%$ efficiency.

\section{SCENARIOS FOR OPTIMAL ICE WARMUP}

During warmup of the ICE, friction losses lower the fuel efficiency of the vehicle. To show the influence of the control model in the EMS, six scenarios are defined, that optimize the vehicle during warmup, using different decision variables, states and constraints:

(A) conventional drive line, defining an upper bound on friction losses;

(B) conventional drive line, defining a lower bound on friction losses;

(C) conventional drive line, fixed cycle;

(D) hybrid drive line, fixed cycle, EMS without optimal warmup;

(E) hybrid drive line, fixed cycle, EMS with optimal warmup;

(F) hybrid drive line, fixed cycle, EMS with optimal warmup and battery constraints.

All scenarios start at cold condition $T\left(t_{0}\right)$, include reaching the nominal temperature $T_{0}\left(t_{1}\right)$ of the warm ICE, and are defined in the following subsections. The simulation results of the scenarios, with the plant model from Section 1, are discussed in Section 4.

\subsection{Conventional, Free Cycle, Upper Bound on Friction Losses (A)}

This scenario aims at finding the drive cycle that maximizes the friction losses during warmup, resulting in the lowest fuel efficiency for warmup. The drive cycle is free, i.e. the load $\left(P_{d}\right)$ and the speed of the vehicle can be freely chosen. Because in this scenario the drive line is conventional $\left(P_{m}=0\right)$, and the GB has a continuously variable ratio with $100 \%$ efficiency, the scenario is equivalent to finding the speed $(\omega)$ and load $\left(P_{e}\right)$ on the ICE, that maximize the friction losses $P_{\text {frc }}$. The cycle ends when $T_{0}$ is reached, i.e. the ICE is warm. The optimization to perform is:

$$
\min _{\left[\omega, P_{e}\right]} \int_{t_{0}}^{t_{1}}-P_{f r c}\left(E_{T}(t), \omega\left(E_{T}\right), P_{e}\left(E_{T}\right)\right) d t
$$

The optimization is numerically solved by iteratively simulating the conventional drive line, until the minimum in (24) is found. The decision variable $\left[\omega, P_{e}\right]$ is thereby parameterized as a function of $E_{T}$ instead of $t$, because $E_{T}\left(t_{1}\right)$ is known a priori, whereas $t_{1}$ is not. The parametrization is implemented as a lookup table, where the number of breakpoints is chosen high enough, to not influence the cost function within a numeric tolerance.

\subsection{Conventional, Free Cycle, Lower Bound on Friction Losses (B)}

This scenario is equal to scenario A, except that the friction losses are minimized instead of maximized:

$$
\min _{\left[\omega, P_{e}\right]} \int_{t_{0}}^{t_{1}} P_{f r c}\left(E_{T}(t), \omega\left(E_{T}\right), P_{e}\left(E_{T}\right)\right) d t
$$

The friction losses of any drive cycle, will fall within the bounds calculated by scenario A and B, as will be shown in Section 4, Figure 17.

\subsection{Conventional, Fixed Cycle (C)}

This scenario defines a conventional vehicle, following a prescribed drive cycle. The drive cycle under consideration is the FTP-75, which is a urban cycle for light-duty vehicles. This cycle is converted to a load cycle $P_{d}$ for a heavy-duty vehicle, as described in Appendix A. The length of the cycle $t_{c}$ is such that $t_{0}<t_{1}<t_{c}$, i.e. the ICE is warm within the cycle. The GB is assumed to have a continuously variable ratio, and to facilitate EMS analysis, the ratio is chosen to keep the ICE at a constant $\omega=1300 \mathrm{rpm}$, which is the lowest possible constant speed to track $P_{d}$. In this scenario, no control freedom is left, and the fuel consumption can be directly evaluated:

$$
\int_{t_{0}}^{t_{c}} P_{f}\left(E_{T}(t), P_{d}(t)\right) d t
$$

\subsection{Hybrid, Fixed Cycle, No Optimal Warmup (D)}

The HEV has additional control freedom compared to the conventional vehicle: the EMS controls the power split with $P_{b}$ and the stop-start functionality with $i_{f}$. The energy in the battery $E_{b}\left(t_{c}\right)$ is equal to $E_{b}\left(t_{0}\right)$, so that the battery is used as a buffer, and not as an energy source. In this scenario, $E_{b}$ is not constrained. In the control model, the thermal state $E_{T}$ is ignored, causing a deliberate mismatch between control model and plant model:

$$
\begin{array}{ll} 
& \min _{\left[P_{b}, i_{f}\right]} \int_{t_{0}}^{t_{c}} P_{f}\left(E_{b}(t), P_{b}(t), i_{f}(t), P_{d}(t)\right) d t \\
\text { s.t. } & \\
& E_{b}\left(t_{0}\right)-E_{b}\left(t_{c}\right)=\Delta E_{b}=0
\end{array}
$$

The optimization is solved using the optimal control framework, as explained in Section 3. 


\subsection{Hybrid, Fixed Cycle, Optimal Warmup (E)}

This scenario is equal to the previous one, except that $E_{T}$ is explicitly taken into account in the control model. The control model equals the plant model:

$$
\min _{\left[P_{b}, i_{f}\right]} \int_{t_{0}}^{t_{c}} P_{f}\left(E_{b}(t), E_{T}(t), P_{b}(t), i_{f}(t), P_{d}(t)\right) d t
$$

s.t.

$$
\begin{aligned}
& \Delta E_{b}=0 \\
& T(t)-T_{0} \leq 0
\end{aligned}
$$

\subsection{Hybrid, Fixed Cycle, Optimal Warmup with Battery Constraints (F)}

This scenario is equal to the previous one, except that constraints on $E_{b}$ are enforced, both upper $\left(\overline{E_{b}}\right)$ and lower $\left(\underline{E_{b}}\right)$ bound:

$$
\min _{\left[P_{b}, i_{f}\right]} \int_{t_{0}}^{t_{c}} P_{f}\left(E_{b}(t), E_{T}(t), P_{b}(t), i_{f}(t), P_{d}(t)\right) d t
$$

s.t.

$$
\begin{aligned}
& \Delta E_{b}=0 \\
& E_{b}(t)-\overline{E_{b}} \leq 0 \\
& \frac{E_{b}}{\bar{T}}-E_{b}(t) \leq 0 \\
& T(t)-T_{0} \leq 0
\end{aligned}
$$

An overview of the optimizations in scenarios $A$ to $F$, is given by (30) with Table 2:

$$
\begin{array}{cc}
\min _{u} J(u, t)= & \min _{u} \int_{t_{0}}^{t_{f}} g(x(t), u(t), w(t)) d t \\
\text { s.t. } & \\
& h_{0}=0 \\
& h_{1,2} \leq 0
\end{array}
$$

TABLE 2

ICE warmup scenarios summarized

\begin{tabular}{c|c|c|c|c|c|c}
\hline & $\mathrm{A}$ & $\mathrm{B}$ & $\mathrm{C}$ & $\mathrm{D}$ & $\mathrm{E}$ & $\mathrm{F}$ \\
\hline$g$ & $-P_{f r c}$ & $P_{f r c}$ & $P_{f}$ & $P_{f}$ & $P_{f}$ & $P_{f}$ \\
\hline$x$ & $E_{T}$ & $E_{T}$ & - & $E_{b}$ & {$\left[\begin{array}{c}E_{b} \\
E_{T}\end{array}\right]$} & {$\left[\begin{array}{c}E_{b} \\
E_{T}\end{array}\right]$} \\
\hline$t_{f}$ & $t_{1}$ & $t_{1}$ & $t_{c}$ & $t_{c}$ & $t_{c}$ & $t_{c}$ \\
\hline$u$ & {$\left[\begin{array}{c}\omega \\
P_{e}\end{array}\right]$} & {$\left[\begin{array}{c}\omega \\
P_{e}\end{array}\right]$} & - & {$\left[\begin{array}{c}P_{b} \\
i_{f}\end{array}\right]$} & {$\left[\begin{array}{c}P_{b} \\
i_{f}\end{array}\right]$} & {$\left[\begin{array}{c}P_{b} \\
i_{f}\end{array}\right]$} \\
\hline$w$ & - & - & $P_{d}$ & $P_{d}$ & $P_{d}$ & $P_{d}$ \\
\hline$h_{0}$ & - & - & - & $\Delta E_{b}$ & $\Delta E_{b}$ & $\Delta E_{b}$ \\
\hline$h_{2}$ & - & - & - & - & $T-T_{0}$ & $T-T_{0}$ \\
\hline$h_{1}$ & - & - & - & - & - & $\left\{\begin{array}{c}E_{b}-\overline{E_{b}} \\
\underline{E_{b}}-E_{b}\end{array}\right.$ \\
\hline
\end{tabular}

with the cost function $J$ as a function of states $x$, decision variables $u$, disturbance $w$, subject to constraints $h$.

\section{OPTIMAL CONTROL PROBLEM SOLUTION}

Scenario $\mathrm{F}$ defines a dynamic optimization problem, with pure state constraints on two states, and is non-autonomous, because the cost function depends explicitly on time, due to disturbance $w(t)=P_{d}(t)$. This problem can be solved directly, e.g. with dynamic programming, or indirectly using e.g. optimal control [23]. The latter is opted for, because it has the potential of being computationally efficient, thus facilitating real-time implementation. Furthermore it provides insight in the optimal solution, as will be shown in Section 4. By solving scenario F, the same methods can be applied to solve scenarios D and E.

In Section 3.1, we summarize necessary conditions for the optimal control problem. In Sections 3.2 to 3.5, these conditions are applied to scenario $\mathrm{F}$, where subsequently the continuous costate dynamics, explicit Hamiltonian minimization, costate jump conditions and solving for the initial costates, are described.

\subsection{Optimal Control Conditions}

Following [8], the cost functional $J$ in (30), with the state equations given by:

$$
\dot{x}=f(x(t), u(t))
$$

lead to the Hamiltonian:

$$
H=g(x(t), u(t), w(t))+\lambda^{T} f(x(t), u(t))
$$

which is, for the optimal solution, always equal or smaller than the Hamiltonian of any other trajectory:

$$
H\left(x^{*}(t), u^{*}(t), \lambda^{*}(t), t\right) \leq H\left(x^{*}(t), u(t), \lambda^{*}(t), t\right)
$$

where the superscript * denotes the optimal solution. The (Euler-Lagrange) necessary conditions are:

$$
\begin{gathered}
\dot{x}^{*}(t)=\frac{\partial H}{\partial \lambda} \\
\dot{\lambda}^{*}(t)=-\frac{\partial H}{\partial x}
\end{gathered}
$$

For the boundary conditions, additional transversality conditions hold, and are given (omitting the penalty on the final state) by:

$$
\left[-\lambda^{*}\left(t_{f}\right)\right]^{T} \delta x_{f}+\left[H\left(x^{*}\left(t_{f}\right), u^{*}\left(t_{f}\right), \lambda^{*}\left(t_{f}\right), t_{f}\right)\right] \delta t_{f}=0
$$


If $t_{f}$ and $x\left(t_{f}\right)$ are specified, the variation of $\delta x_{f}$ and $\delta t_{f}$ in (36) is 0 , and the transversality condition reduces to:

$$
\begin{aligned}
& x^{*}\left(t_{f}\right)=x_{f} \\
& x^{*}\left(t_{0}\right)=x_{0}
\end{aligned}
$$

When the state is on the constraint within $\left(t_{0}, t_{f}\right)$, interior boundary conditions apply [9]:

$$
\begin{gathered}
H^{+}\left(t_{j}\right)=H^{-}\left(t_{j}\right)-\zeta\left(t_{j}\right) \frac{\partial h\left(t_{j}\right)}{\partial t} \\
\lambda^{+}\left(t_{j}\right)=\lambda^{-}\left(t_{j}\right)+\zeta\left(t_{j}\right) \frac{\partial h\left(t_{j}\right)}{\partial x}
\end{gathered}
$$

where the superscript ${ }^{-}$and ${ }^{+}$denote the left-hand side and right-hand side limit values respectively, at $t_{j}$. The conditions describe a possible jump condition in $\lambda$ and $H$ at $t_{j}$, when the state makes contact with the boundary $h$, see Appendix B. The magnitude of the jump, is determined by the parameter $\zeta$, and is, together with the number of jumps, not known $a$ priori. From (38) and (39) we can derive the following lemmas:

Lemma 1: When the constraint $h$ at jump $j$ is time invariant (i.e. $\frac{\partial h\left(t_{j}\right)}{\partial t}=0$ ), $H$ is continuous at the junction.

Lemma 2: When $h$ does not have mixed terms in $x$, a jump in the costate due to an active constraint, does not cause a jump in the costate of the state without active constraints. Consequently, the costate dynamics of the state without active constraints, are continuous.

\subsection{Costate Dynamics}

To improve readability, the dependency on time is omitted in the remainder of this paper. The system dynamics are given by:

$$
f=\left[\begin{array}{c}
\dot{x}_{1} \\
\dot{x}_{2}
\end{array}\right]=\left[\begin{array}{c}
\dot{E}_{b} \\
\dot{E}_{T}
\end{array}\right]=\left[\begin{array}{c}
-P_{b i} \\
-P_{T}
\end{array}\right]
$$

and using (29), results in the Hamiltonian $H$ :

$$
H=P_{f}-\left[\begin{array}{ll}
\lambda_{1} & \lambda_{2}
\end{array}\right]\left[\begin{array}{l}
P_{b i} \\
P_{T}
\end{array}\right]
$$

To derive the dynamics of the costates (35), we can evaluate the costates separately, as there are no mixed terms in $\frac{\partial H}{\partial x}$. The first costate $\lambda_{1}$ is constant, using $(19,20$, $35)$ and (41):

$$
\dot{\lambda}_{1}=-\frac{\partial H}{\partial E_{b}}=\lambda_{1} \frac{\partial P_{b i}}{\partial E_{b}}=0
$$

Only when constraint $h_{1}$ becomes active, $\lambda_{1}$ jumps and becomes piecewise constant. The jump conditions are treated in Section 3.4.

The second costate $\lambda_{2}$ dynamics are derived separately, for all combinations of $i_{t h}$ and $i_{f}$.

If $i_{t h}=0$ (thermostat is off) and $i_{f}=1$ (ICE is running), then the dynamics are derived using $(2,15,16,35,41)$ :

$$
\dot{\lambda}_{2}=-\frac{\partial H}{\partial E_{T}}=-\frac{\partial P_{f}}{C \partial T}+\lambda_{2} \frac{\partial P_{T}}{C \partial T}
$$

Substituting the partial derivatives of (11):

$$
\frac{\partial P_{f}}{\partial T}=-\frac{1}{\eta} \frac{\partial P_{f r c}}{\partial T}
$$

and (17) with $i_{t h}=P_{h}=P_{h 0}=0$ :

$$
\frac{\partial P_{T}}{\partial T}=\frac{-k \partial P_{f}}{\partial T}+\frac{\partial P_{f r c}}{\partial T}=\left(\frac{\kappa}{\eta}+1\right) \frac{\partial P_{f r c}}{\partial T}
$$

into (43), results in:

$$
\dot{\lambda}_{2}=\frac{1}{C}\left(\frac{1}{\eta}+\lambda_{2}+\frac{\lambda_{2} k}{\eta}\right) \frac{\partial P_{f r c}}{\partial T}
$$

with:

$$
\frac{\partial P_{f r c}}{\partial T}=-\alpha P_{f r 0}(\omega) \gamma e^{-\alpha\left(T-T_{0}\right)}
$$

If $i_{t h}=0$ and $i_{f}=0$, then the ICE is stopped and disconnected, thus resulting in $\dot{\lambda}_{2}=0$, and $\lambda_{2}$ is constant.

If $i_{\text {th }}=1$, (i.e. an activated thermostat with $T=T_{0}$,) and $P_{h}=P_{h 0}$, then from (17), $P_{t h}=0$ and $\lambda_{2} P_{t h}$ in (41) is zero, for both $i_{f}=0$ and $i_{f}=1$.

\subsection{Explicit Solution of the Hamiltonian}

The minimization of $H$ in (33) can be solved explicitly as a function of $\left(P_{d}, \lambda_{1}, \lambda_{2}\right)$ and the model parameters. The method in [10] is here extended to include a second (co)state, related to the thermal dynamics of the ICE. The solution to the minimization is characterized by four operating regions (modes), dependent on the stop-start command $i_{f}$ and the sign of $P_{b}$ :

- $M_{m}$ : 'MG only', the drive train is using only the MG, with the ICE decoupled and stopped $\left(i_{f}=0\right)$;

$-M_{i}$ : 'ICE only', the drive train is using only the ICE, and $P_{b}=0$

- $M_{b}$ : 'boost', both MG and ICE provide positive power to the drive train, $P_{b}>0$;

- $M_{c}$ : 'charge', the MG withdraws power from the drive train to charge the battery $\left(P_{b}<0\right)$, while the ICE provides positive power. 
TABLE 3

Explicit solution to the Hamiltonian

\begin{tabular}{c|c|c}
\hline Mode & $P_{b}^{*}$ & $i_{f}^{*}$ \\
\hline$M_{m}$ & $\frac{P_{d}}{\eta_{m}}$ & 0 \\
\hline$M_{i}$ & 0 & 1 \\
\hline$M_{b}$ & $-\frac{\left(\lambda_{1}+\frac{\eta_{m}}{\eta} L\right)}{\left(2 \beta \lambda_{1}\right)}$ & 1 \\
\hline$M_{c}$ & $-\frac{\left(\lambda_{1}+\frac{1}{\eta \eta_{m}} L\right)}{\left(2 \beta \lambda_{1}\right)}$ & 1 \\
\hline
\end{tabular}

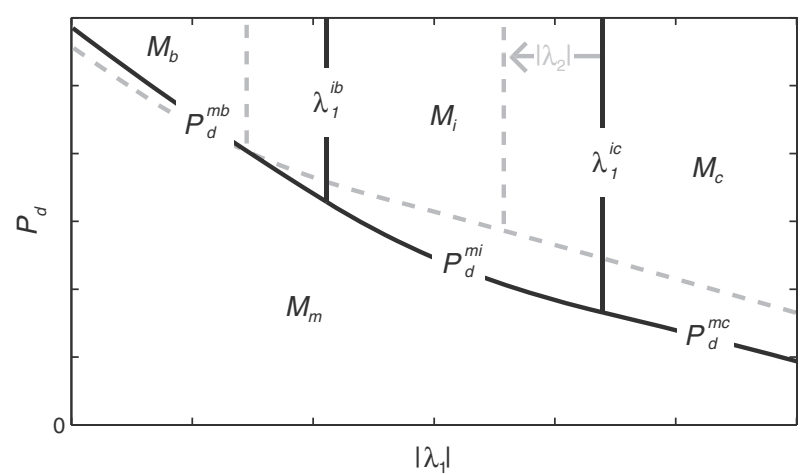

Figure 8

Schematic control map, indicating the guards $\left\{\lambda_{1}^{i b}, \lambda_{1}^{i c}, P_{d}^{m b}, P_{d}^{m i}, P_{d}^{m c}\right\}$ of the modes $\left\{M_{m}, M_{i}, M_{b}, M_{c}\right\}$. At low temperatures the guards change as a function of $\lambda_{2}$ and $P_{f r}$, shown by the grey dotted lines.

For each mode, the optimal control signal is calculated by solving $\frac{d H}{d u}=0$ for $u$, Table 3 . Note that for $M_{b}$ and $M_{c}, P_{b}^{*}$ is not a function of $P_{d}$.

All modes have an associated Hamiltonian, of which only one is minimal for a certain region $\left(P_{d}, \lambda_{1}, \lambda_{2}\right)$, separated by switching surfaces (guards). The guards are calculated by equating the Hamiltonians of two neighboring modes, and solving for $\lambda_{1}$ or $P_{d}$. The guards between two modes are depicted with the superscripts $\{m, i, b, c\}$ and are schematically depicted in Figure 8 , as a function of $\lambda_{1}$ and $P_{d}$. Two guards are a function of $\left(\lambda_{1}, \lambda_{2}\right)$ :

$$
\begin{aligned}
& \lambda_{1}^{i b}=-\frac{\eta_{m} L}{\eta} \\
& \lambda_{1}^{i c}=-\frac{L}{\eta \eta_{m}}
\end{aligned}
$$

with:

$$
L=1+\kappa \lambda_{2}
$$

Three guards are a function of $\left(P_{d}, \lambda_{1}, \lambda_{2}\right)$ :

$$
\begin{gathered}
P_{d}^{m b}=-\frac{\eta \lambda_{1}+\eta_{m} L}{2 \beta \lambda_{1} \frac{\eta}{\eta_{m}}}-\frac{\sqrt{L\left(4 \beta \eta \lambda_{1} P_{f r}\right)}}{2 \beta \lambda_{1} \frac{\eta}{\eta_{m}}} \\
P_{d}^{m i}=-\frac{\eta \lambda_{1}+\eta_{m} L}{2 \beta \lambda_{1} \frac{\eta}{\eta_{m}}} \\
-\frac{\sqrt{L\left(4 \beta \eta \lambda_{1} P_{f r}+2 \lambda_{1} \eta \eta_{m}+L \eta_{m}^{2}\right)+\left(\lambda_{1} \eta\right)^{2}}}{2 \beta \lambda_{1} \frac{\eta}{\eta_{m}}} \\
P_{d}^{m c}=-\frac{\eta \lambda_{1}+\eta_{m} L}{2 \beta \lambda_{1} \frac{\eta}{\eta_{m}}} \\
-\frac{\sqrt{L\left(4 \beta \eta \lambda_{1} P_{f r}+2 \lambda_{1} \eta\left(\eta_{m}-\eta_{m}^{-1}\right)+L\left(\eta_{m}^{2}-\eta_{m}^{-2}\right)\right)}}{2 \beta \lambda_{1} \frac{\eta}{\eta_{m}}}
\end{gathered}
$$

All modes are explicitly defined with (48-53), as a function of $\left(P_{d}, \lambda_{1}, \lambda_{2}\right)$ and the model parameters, shown in Table 5.

The minimization of $\mathrm{H}$ is now efficiently solved in three steps for given $\left(P_{d}, \lambda_{1}, \lambda_{2}\right)$ :

(1) for each mode, calculate $P_{b}^{*}$;

(2) determine the optimal mode, using (48-53);

(3) for the mode found, select $P_{b}^{*}$ and $i_{f}^{*}$.

With $\lambda_{2}$ decreasing at low temperatures, the guards shift, as schematically depicted in Figure 8, but also change shape, as can be determined from the dependency on $L\left(\lambda_{2}\right)$ and $P_{f r}(T)$, in (51-53) and (7). As a result, for the same $\lambda_{1}$, the load on the ICE typically increases at low temperatures, due to the larger $M_{m}$ area (i.e. only higher loads are performed with the ICE) and the shift from $M_{b}$ and $M_{i}$ towards $M_{c}$ (i.e. an increasing tendency to charging with the ICE). Note that power limits of the components are easily added to the control map, and, contrarily to [10], the control map is a function of $\lambda_{2}$ due to the additional state, but it is identical when $\lambda_{2}=0$.

\subsection{Jumps in the Costates}

The states are constrained by $h_{1}$ and $h_{2}$ as defined in (30) with Table 2. For these constraints, Lemma 2 holds, and the jump condition (39), can be analyzed for each costate separately.

For $\lambda_{1}$, jumps occur at times $t_{k}$, when constraint $h_{1}$ is active:

$$
\lambda_{1}^{+}\left(t_{k}\right)=\lambda_{1}^{-}\left(t_{k}\right)+\zeta\left(t_{k}\right) \frac{\partial h_{1}\left(t_{k}\right)}{\partial E_{b}}
$$

resulting in a piece-wise constant $\lambda_{1}$. A priori it is not possible to determine the amount of jumps, nor times $t_{k}$, 
nor the values of $\zeta$. To solve this problem, the iterative procedure from [11] is adopted, which determines $\lambda_{1}^{+}\left(t_{k}\right)$ $(k=\{2, . ., n\})$ for $n$ number of jumps, without evaluating $\zeta$. See Section 3.5 for an outline of the procedure.

For $\lambda_{2}$, a jump occurs at $t_{1}$, when constraint $h_{2}$ is active. Next, we will show that $\lambda_{2}^{-}\left(t_{1}\right)=0$, which facilitates finding $\lambda_{2}\left(t_{0}\right)^{*}$. At $t_{1}^{+}$, the thermostat is active $\left(i_{t h}=1\right)$ and $P_{T}=0$ (17), resulting in $H^{+}=P_{f}^{+}-\lambda_{1} P_{b i}^{+}$with $\lambda_{2}^{+}$undefined. Therefore, (39) can not be used. The value of $\lambda_{2}^{-}$however can be calculated using the explicit solution of $H$ and the jump condition (38). When we have at $t_{1}$ :

$-\lambda_{1}^{-}=\lambda_{1}^{+}=\lambda_{1}$ (no active battery limit),

$-P_{d}^{-}=P_{d}^{+}=P_{d}\left(P_{d}\right.$ is continuous $)$,

$-i_{t h}^{-}=0$ (thermostat not active),

$-i_{t h}^{+}=1$ (thermostat is active),

$-\frac{\partial h\left(t_{1}\right)}{\partial t}=0$ (constraint independent on time), then (38) can be rewritten as:

$$
\begin{aligned}
H^{-} & =H^{+} \Longleftrightarrow \\
P_{f}^{-}-\lambda_{1} P_{b i}^{-}-\lambda_{2}^{-} P_{T}^{-} & =P_{f}^{+}-\lambda_{1} P_{b i}^{+}
\end{aligned}
$$

Considering all possible mode transitions for $\mathrm{H}^{-}$and $\mathrm{H}^{+}$, we end up with $(4 \cdot 4=) 16$ mode combinations as indicated in Table 4. Therein $M_{m}^{-}$is not viable, as for $M_{m}: P_{T}=0$ and $T_{0}$ is never reached. For all other mode transitions, the explicit solution for $u$ (Tab. 3) is used to solve (55) for $\lambda_{2}^{-}$. All candidate solutions of $\lambda_{2}^{-}$are evaluated on their optimality by using (33) and the model parameters from Table 5. The result from this analysis is summarized in Table 4 as:

- ' 0 ' indicating a mode transition, where the optimal $\lambda_{2}^{-}=0$;

- '-' indicating a mode transition, which is never optimal;

- ' 0 ' ' indicating a mode transition, which is only optimal on the guard of the two modes. Then $\lambda_{2}^{-}=0$.

These results show, that for all optimal solutions: $\lambda_{2}^{-}\left(t_{1}\right)=0$, and that a mode change at $t_{1}$ is only optimal, when exactly on a guard. This result is different from [7] and [14], where $h_{2}$ is not present and $\lambda_{2}\left(t_{f}\right)=0$. Note that $\dot{\lambda}_{2}^{-}\left(t_{1}\right) \neq 0$ due to (46).

TABLE 4

Solutions for $\lambda_{2}^{-}\left(t_{1}\right)$ for all mode transitions from $M^{-}$to $M^{+}$

\begin{tabular}{c|c|c|c|c}
\hline & $M_{i}^{+}$ & $M_{b}^{+}$ & $M_{c}^{+}$ & $M_{m}^{+}$ \\
\hline$M_{i}^{-}$ & 0 & $0^{\prime}$ & $0^{\prime}$ & $0^{\prime}$ \\
\hline$M_{b}^{-}$ & $0^{\prime}$ & 0 & - & $0^{\prime}$ \\
\hline$M_{c}^{-}$ & $0^{\prime}$ & - & 0 & $0^{\prime}$ \\
\hline$M_{m}^{-}$ & - & - & - & - \\
\hline
\end{tabular}

\subsection{Solving the Two-Point-Boundary Value Problem}

The dynamic, non-autonomous, constrained two-state problem is solved explicitly, with two nested optimizations remaining:

- inner loop: find $\lambda_{2}\left(t_{0}\right)$ that results in $\lambda_{2}\left(t_{1}\right)=0$;

- outer loop: find $\lambda_{1}\left(t_{0}\right)$ that results in $E_{b}\left(t_{0}\right)=E_{b}\left(t_{f}\right)$.

Both loops are solved over the drive cycle, with the gradient descent method, until $\lambda_{1}\left(t_{0}\right)$ and $\lambda_{2}\left(t_{0}\right)$ have an accuracy $>1 \mathrm{e}-4$. The remaining error on $\Delta E_{b}$ is checked to be less than $1 \%$, and corrected in the fuel consumption, with the cycle averaged $\lambda_{1}$. The inner loop needs typically 3 iterations to converge, the outer loop 8 iterations.

If also constraints on $E_{b}$ are considered, then the method from [11] is implemented in the outer loop, where for $n$ constraint violations, additional parameters $\lambda_{1}^{+}\left(t_{k}\right)$ are found. This optimization is started with the unconstrained problem, and the time step $\left(t_{k}\right)$ of the largest constraint violation of $E_{b}$ is recorded. In this first iteration, $t_{k-1}$ is set at $t_{0}$ and $t_{k+1}$ is set at $t_{f}$. The problem is split in two segments, where $E_{b}\left(t_{k}\right)$ is set at the constraint, with the first segment from $t_{k-1}$ to $t_{k}$, and the second segment from $t_{k}$ to $t_{k+1}$. Both segments are independently solved for $\lambda_{1}^{+}\left(t_{k-1}\right)$ and $\lambda_{1}^{+}\left(t_{k}\right)$. This bi-section process is repeated, until all segments are lacking constraint violations, resulting in the piece-wise constant $\lambda_{1}^{*}$.

\section{SIMULATION RESULTS}

In this section simulation results of scenarios A to F, defined in Section 1, with the parameters from Table 5, are presented, showing the differences in ICE warming speed,

TABLE 5

Model parameters for simulation

\begin{tabular}{c|c|c|c}
\hline & Value & Unit & Description \\
\hline$\eta$ & 0.5 & - & Indicated efficiency \\
\hline$\kappa$ & 0.25 & - & Fuel heat fraction into coolant \\
\hline$T_{0}$ & 85 & ${ }^{\circ} \mathrm{C}$ & Nominal temperature \\
\hline$P_{0}$ & $-2 \mathrm{e} 4$ & $\mathrm{~W}$ & Nominal engine friction at $\omega_{0}$ \\
\hline$\omega_{0}$ & 1300 & $\mathrm{rpm}$ & Nominal engine speed \\
\hline$C$ & $6 \mathrm{e} 5$ & $\mathrm{~J} /{ }^{\circ} \mathrm{C}$ & Heat capacity \\
\hline$\theta$ & 30 & $\mathrm{~W} /{ }^{\circ} \mathrm{C}$ & Heat transfer coefficient \\
\hline$\gamma$ & 0.1 & - & Viscous friction fraction \\
\hline$\alpha$ & $3.6 \mathrm{e}-2$ & $1 /{ }^{\circ} \mathrm{C}$ & Empirical viscosity constant \\
\hline$\eta_{m}$ & 0.9 & - & Mechanical efficiency MG \\
\hline$\beta$ & $6 \mathrm{e}-7$ & $1 / \mathrm{W}$ & Loss coefficient battery \\
\hline
\end{tabular}


TABLE 6

Simulation results for the warmup scenarios, with $T\left(t_{0}\right)=-10^{\circ} \mathrm{C} . E_{f 0}$ is $E_{f}$ for $T\left(t_{0}\right)=T_{0}=85^{\circ} \mathrm{C}$

\begin{tabular}{l|c|c|c|c|c|c}
\hline & $\mathrm{A}$ & $\mathrm{B}$ & $\mathrm{C}$ & $\mathrm{D}$ & $\mathrm{E}$ & $\mathrm{F}$ \\
\hline$E_{d}(\mathrm{MJ})$ & 0 & 155.6 & 107.2 & 107.2 & 107.2 & 107.2 \\
\hline$E_{f}(\mathrm{MJ})$ & 152 & 213 & 289.2 & 217.3 & 216.3 & 216.3 \\
\hline$E_{f r c}(\mathrm{MJ})$ & 18.9 & 3.8 & 11.2 & 6.30 & 5.02 & 5.04 \\
\hline$t_{1}(\mathrm{~s})$ & 7186 & 389 & 1174 & 1736 & 1785 & 1785 \\
\hline$\Sigma t_{t h}(\mathrm{~s})$ & 7186 & 389 & 1174 & 531 & 478 & 478 \\
\hline$\left|\lambda_{1}\right|(-)$ & - & - & - & 2.18 & 2.15 & $\left\{\begin{array}{l}2.14 \\
2.16\end{array}\right.$ \\
\hline$\left|\lambda_{2}\right|(-)$ & - & - & - & - & 0.552 & 0.553 \\
\hline$E_{f 0}(\mathrm{MJ})$ & - & - & 270.5 & 204.7 & 204.7 & 204.7 \\
\hline
\end{tabular}

the friction losses and the principle behavior of the optimal control solution, with and without constraints. The scenarios in Section 4.1 start with $T\left(t_{0}\right)=-10^{\circ} \mathrm{C}$, and in Section 4.2 the initial temperature is varied. The results are summarized in Table 6 and explained next. Note that all $\lambda \leq 0$ and referred to with the absolute value $|\lambda|$.

\subsection{Scenario A-F, Low Initial Temperature}

Scenario A is the most costly ICE warmup strategy. The optimal solution shows no dependency on $E_{T}$ : the ICE is running constantly at low idle $(\omega=550 \mathrm{rpm})$, without performing mechanical work $\left(E_{d}=0\right)$ :

$$
E_{d}=\int_{t_{0}}^{t_{f}} P_{d} d t
$$

As the fuel flow is very low, the warmup time $t_{1}$ is very long: $7186 \mathrm{~s}$, and the total amount of cold friction losses $E_{f r c}$ is maximal: $18.9 \mathrm{MJ}$, with a considerable total fuel consumption $\left(E_{f}=152 \mathrm{MJ}\right)$ :

$$
E_{f}=\int_{t_{0}}^{t_{f}} P_{f} d t
$$

We define the total time the ICE is running $\left(i_{f}=1\right)$ during warmup as:

$$
\Sigma t_{t h}=\int_{t_{0}}^{t_{1}} i_{f} d t
$$

with $t_{1}$ the first time $T\left(t_{1}\right)=T_{0}$. In this scenario, $\Sigma t_{t h}$ is equal to $t_{1}$, i.e. the ICE is never stopped. In Figures 9 and 10 , the time traces of A do not fit the scale of the other scenarios, and are only partially plotted.

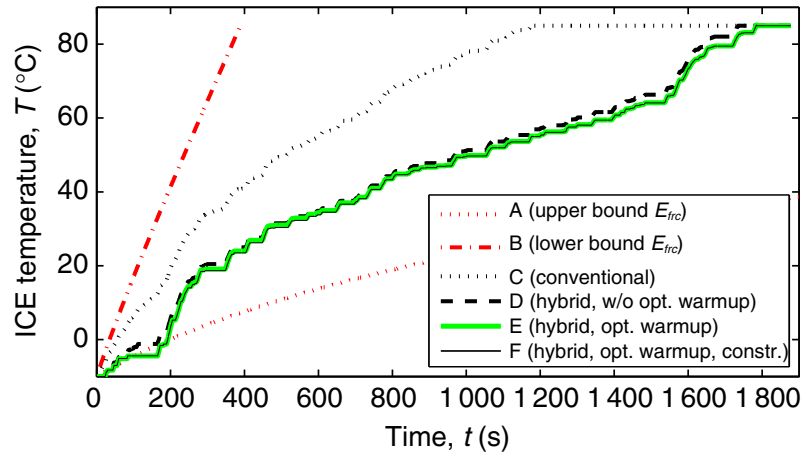

Figure 9

Temperature, during warmup with $T\left(t_{0}\right)=-10^{\circ} \mathrm{C}$.

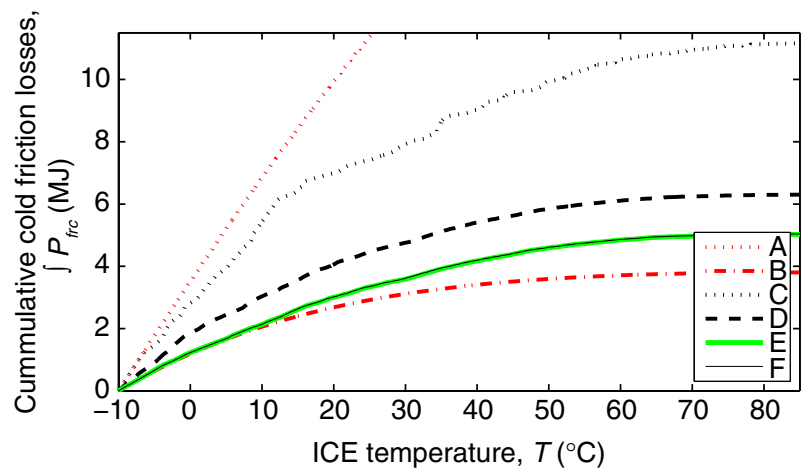

Figure 10

Cumulative cold friction losses during warmup, with $T\left(t_{0}\right)=-10^{\circ} \mathrm{C}$.

Scenario B poses the lower bound on $E_{\text {frc }}=3.8 \mathrm{MJ}$, when the drive cycle can be freely chosen. The optimal solution shows no dependency on $E_{T}$ : the ICE is running at full load at a reasonably low engine speed. That speed is for our engine defined by a corner in the maximum fueling at $\omega=1000 \mathrm{rpm}$, as shown in Figure 3. Whereas scenarios $\mathrm{A}$ and B pose bounds on $E_{f r c}$ as a function of temperature (Fig. 10), Figure 9 shows that scenarios with stop-start (D-F) are not necessarily bounded by A and B over time, e.g. at $t=[100,200] \mathrm{s}$.

For the remainder of the scenarios (C-F), the FTP75 is used as a typical load case for $P_{d}$, as defined in Appendix A.

Scenario $C$ has no control freedom. This scenario shows reasonable fast $t_{1}$, but at the cost of high $E_{f r c}=11.2$ MJ. In the FTP75, a large amount of stops is present, causing the ICE to idle. As idling is not beneficial for efficient warmup, the fuel consumption $E_{f}$ is high. 


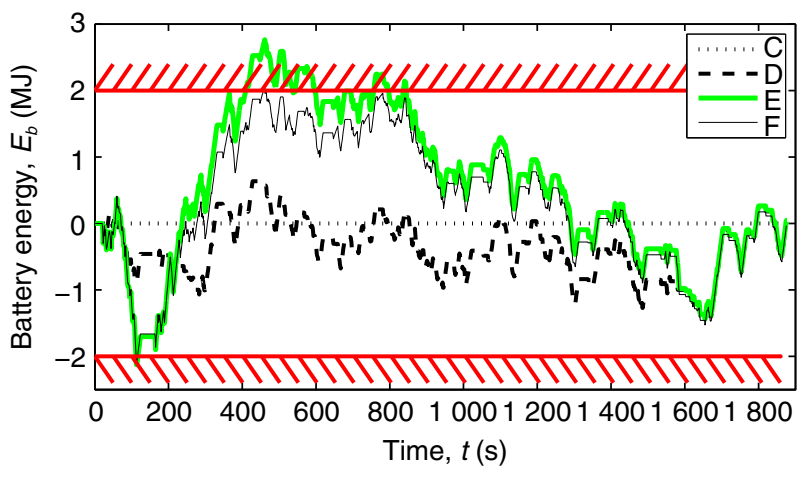

Figure 11

Battery energy, with $T\left(t_{0}\right)=-10^{\circ} \mathrm{C}$. In scenario $\mathrm{F}$, the $E_{b}$ limits at $\pm 2 \mathrm{MJ}$ (red constraint lines) are touched at $t=\{114,461,781\} \mathrm{s}$.

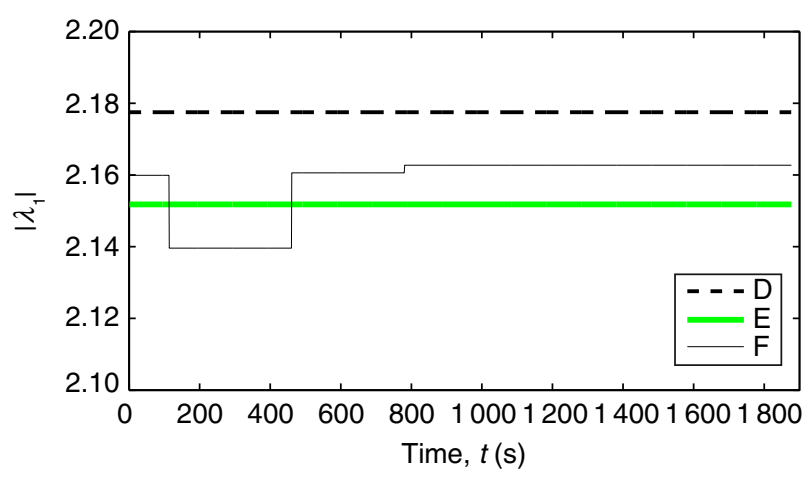

Figure 12

Constant $\lambda_{1}^{*}$ for scenario D and E, and piecewise constant for scenario F, with jumps at $t=\{114,461,781\}$ s, where $E_{b}$ limits are touched.

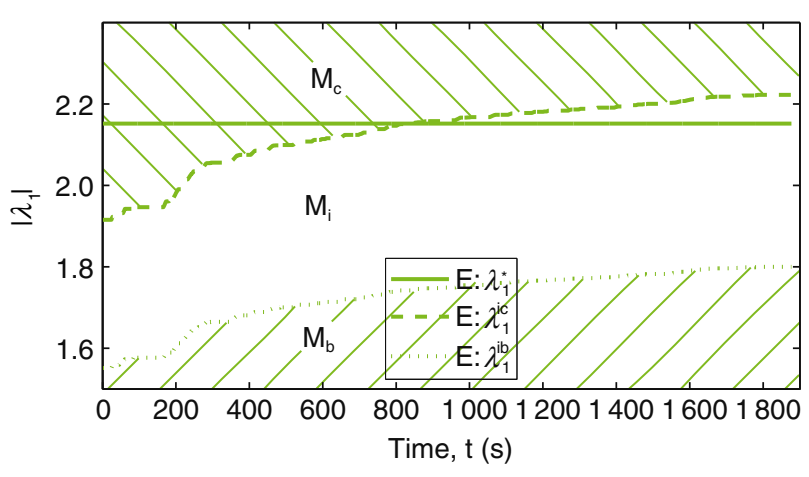

Figure 13

Guards $\lambda_{1}^{x}$ (with $x \in\{i b, i c\}$ ) and mode areas of the control map as a function of $\left(\lambda_{1}, t\right)$, for scenario E. Scenario E starts in $M_{c}$ and switches to $M_{i}$ at $t=847 \mathrm{~s}$, while in scenario $\mathrm{D}, M_{c}$ is never used.

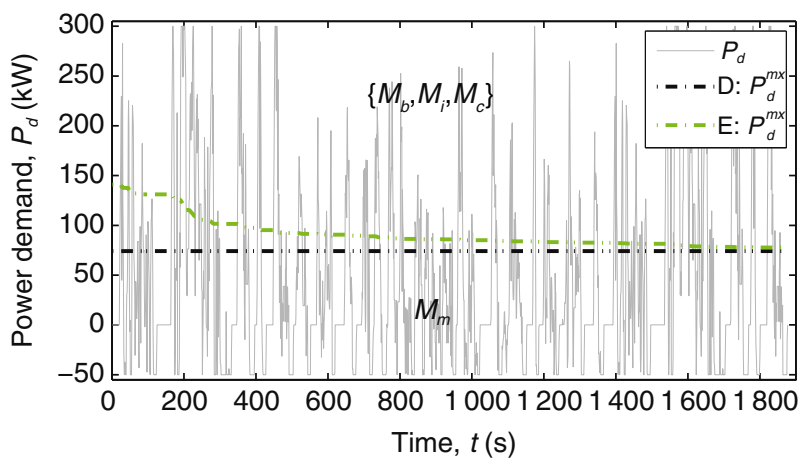

Figure 14

Guards $P_{d}^{m x}$ (with $x \in\{b, i, c\}$ ) of the control map as a function of $P_{d}$, for scenario $\mathrm{D}$ and $\mathrm{E}$. Below this guard, $M_{m}$ ('MG only') is active, above this guard $M_{b}, M_{i}$, or $M_{c}$ is active, dependent on $\lambda_{1}$.
Scenarios D in Figures 9 and 10 show a later $t_{1}$ and much lower $E_{f r c}$ than C, caused by the hybrid system with stopstart, resulting in $\Sigma t_{t h}<t_{1}$. In the temperature trace, the stop periods are clearly visible as having a constant temperature. The hybrid topology is able to save $24.8 \%$ of fuel, due to brake energy recuperation, and stopping the engine at low loads. The battery usage is balanced over the cycle (Fig. 11), with a constant $\lambda_{1}$ (Fig. 12).

Scenario $\mathrm{E}$ is able to add an additional $0.45 \%$ fuel saving, by incorporation of the thermal and friction behavior of the ICE in the EMS, thereby reducing $E_{f r c}$. The difference between scenario D and E can be explained by changes of the guards in the control map from Figure 8, which are represented in Figures 13 and 14. Scenario D has no $\lambda_{2}$ and a constant $\lambda_{1}$, therefore the guards are constant. This results for the $\lambda_{1}$ in Figure 12 in a mode decision between $M_{i}$ and $M_{m}$. As guard $P_{d}^{m x}$ is constant (Fig. 14), all $P_{d}<P_{d}^{m x}$ results in $M_{m}$ ('MG only') and all $P_{d}>P_{d}^{m x}$ results in $M_{i}$ ('ICE only'). Scenario E includes the thermal state, and the corresponding costate $\lambda_{2}$ can be interpreted as, how beneficial it is for fuel consumption, to increase the temperature of the ICE. That benefit is largest at the start of the cycle, and reduces exponentially towards the warm state, as shown in Figure 15 . Due to the dynamics of $\lambda_{2}$, two mechanisms occur in the control map:

- $P_{d}^{m x}$ increases at low temperatures, i.e. due to higher ICE friction, $M_{i}$ is less beneficial than $M_{m}$ and the ICE operates only at higher loads $\left(>P_{d}^{m x}\right.$, Fig. 14)

- the modes shift towards $M_{c}$, where $\lambda_{1}$ starts in $M_{c}$, crossing $\lambda_{1}^{i c}$ at $t=847 \mathrm{~s}$, and continuing in $M_{i}$ (Fig. 13), i.e. the load on the ICE at low temperatures is increased by charging the battery. 


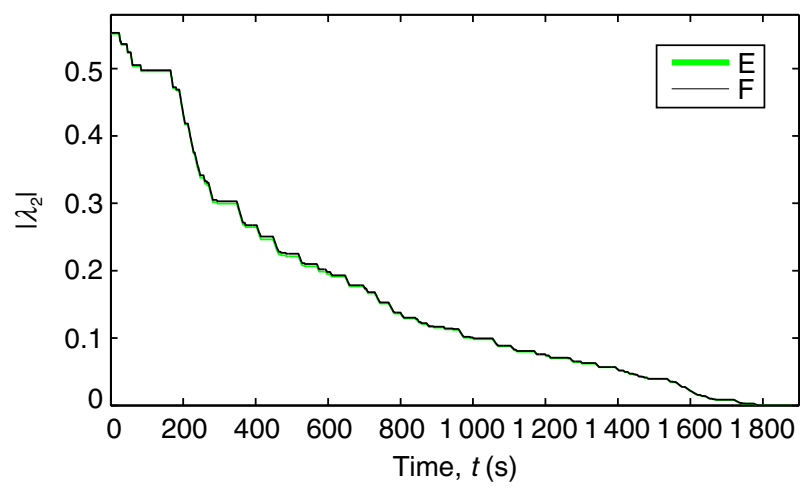

Figure 15

Exponential decrease of $\lambda_{2}^{*}$ for scenario $\mathrm{E}$ and $\mathrm{F}$.

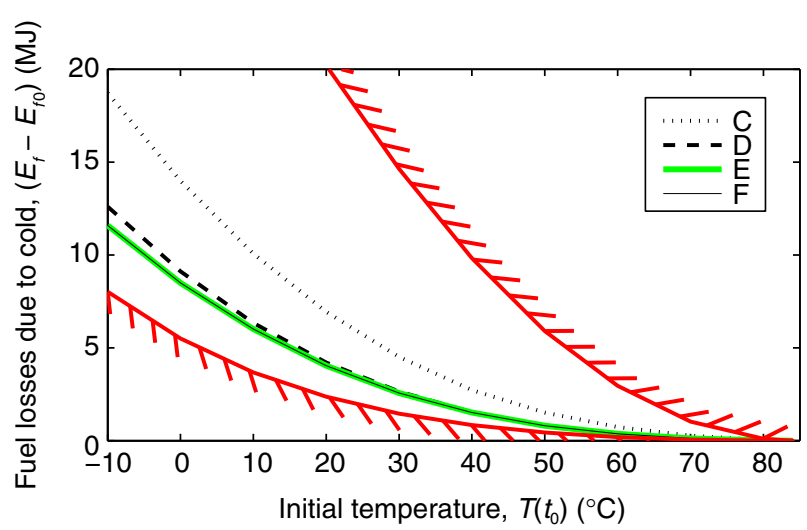

Figure 16

Fuel losses due to warmup, for a range of $T\left(t_{0}\right)$ in scenario $\mathrm{C}$ to $\mathrm{F}$, with the upper and lower bound (in red), as defined by scenario $\mathrm{A}$ and $\mathrm{B}$.

Both mechanisms have an opposite effect on $E_{b}$ and for scenario $\mathrm{E}$ the result is shown in Figure 11 (green line): a high amount of $M_{m}$ at the start of the cycle, depletes the battery, after which the increased charging is prevalent and $E_{b}$ rises until 461 s. After that, $E_{b}$ gradually decreases to zero to ensure charge sustaining operation, mostly in $M_{i}$ and $M_{m}$. The increased load on the ICE reduces $\Sigma t_{t h}$ from 531 to $478 \mathrm{~s}$, whereas $t_{1}$ increases from 1736 to $1785 \mathrm{~s}$ due to the increased amount of $M_{m}$, i.e. longer stop periods of the ICE.

Scenario F shows the influence of battery constraints on the optimal solution. Here upper and lower bounds on $E_{b}$ are enforced. Additional jumps in $\lambda_{1}^{*}$ occur, where $E_{b}$ hits the bound. These jumps occur at $t=114 \mathrm{~s}, t=461 \mathrm{~s}$ and $t=781 \mathrm{~s}$, see Figures 11 and 12 . The jumps in $\lambda_{1}$ of $\mathrm{F}$ (Fig. 12) do not significantly influence the evolution in $\lambda_{2}$ (Fig. 15), as is given by (39). However $\lambda_{2}\left(t_{0}\right)$ must be slightly adjusted for the difference in charging, fueling,

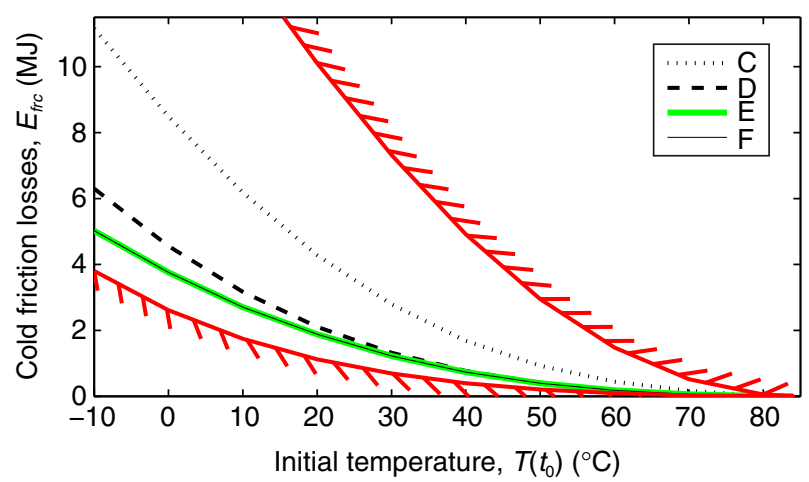

Figure 17

Cold friction losses for a range of $T\left(t_{0}\right)$ in scenario $\mathrm{C}$ to $\mathrm{F}$, with the upper and lower bound (in red), as defined by scenario A and $\mathrm{B}$.

and thus warming, between $\mathrm{E}$ and $\mathrm{F}$. Because of the minor influence of the constraints on $E_{b}$, no significant fuel increase can be seen.

\subsection{Scenario A-F, Varying Initial Temperature}

When $T\left(t_{0}\right)$ increases, the friction losses in the ICE are smaller and, consequently, the fuel benefit of optimal ICE warming will be smaller. The effect of different $T\left(t_{0}\right) \in[-10,85]^{\circ} \mathrm{C}$ on the friction losses is simulated and summarized in Figures 16 and 17 . The nominal fuel consumption when $T\left(t_{0}\right)=T_{0}=85^{\circ} \mathrm{C}$, is denoted as $E_{f 0}$, see also Table 6.

The fuel differences between the scenarios are best explained at $T\left(t_{0}\right)=-10^{\circ} \mathrm{C}$, Figure 16 . In scenario $\mathrm{C}$, the cold friction losses cause a fuel increase of $18.7 \mathrm{MJ}$ ( $+6.9 \%$ ), compared to $E_{f 0}$. For a hybrid vehicle (scenario $\mathrm{D})$, the fuel losses are already much lower (12.6 MJ), where the absolute lower bound (scenario B) is $8 \mathrm{MJ}$. From the 4.6 MJ of fuel difference between $\mathrm{D}$ and $\mathrm{B}$, optimal control (scenario E) is able to save $1 \mathrm{MJ}$, resulting in $11.6 \mathrm{MJ}$ fuel losses.

In Figure 17, the root cause of the losses, $E_{f r c}$, is shown. As $E_{f r c}$ is compensated by the ICE with the combustion of fuel, the following approximate relation holds:

$$
E_{f r c} \approx \eta\left(E_{f}-E_{f 0}\right)
$$

Therefore the trends in Figure 17 are similar to Figure 16, with one notable difference: $E_{f r c}$ of scenarios $\mathrm{E}$ and $\mathrm{F}$ are closer to the lower bound. This is explained by the increased load on the ICE, as seen in Figures 13, 14, which is beneficial for reducing warmup losses. However this is accomplished by increased usage of the battery (Fig. 11), 
which has inherent energy losses, thereby decreasing the total fuel advantage, as shown in Figure 16. Scenario E and $\mathrm{F}$ find the optimal balance between these two counter effects, thereby saving fuel.

If other drive cycles than the FTP are considered, C-F will change, but A and B wil be the same, providing an upper and lower bound of the fuel saving potential of optimal warmup. When the average load of the drive cycle is decreasing, the advantage of optimal warming is increasing. In this section, a heavy duty truck is simulated, which has typical high average load characteristics, and when compared to passenger car applications [7], will have a smaller benefit from optimal warming.

\section{CONCLUSION AND FUTURE WORK}

For the parallel HEV with stop-start, low complexity, control oriented models of ICE, Motor/Generator and battery are developed and validated. The ICE model comprises of a nominal model at warm conditions, corrected with a nonnominal friction model, as a function of the temperature dynamics of the ICE.

Several vehicle scenarios are defined to show the influence of optimal warmup: load cycles defining the upper and lower bound on friction losses, a conventional vehicle running a predefined drive cycle, an HEV on the same cycle, with an EMS ignoring temperature dynamics of the ICE, an HEV with an EMS taking ICE temperature explicitly into account, and an EMS taking additionally the battery constraints explicitly into account.

For the EMS a two-state, non-autonomous, constrained optimal control problem is successfully implemented. The dynamics of the costates, including jump conditions, and the Hamiltonian minimization are explicitly solved. The remaining problem of finding the initial conditions of the costates is solved efficiently for a predetermined drive cycle. For real-time applications using preview information, the developed EMS could be used, which is left for future research.

The EMS is applied to the vehicle scenarios. A HEV with stop-start has clear advantages over a conventional vehicle without stop-start, as e.g. $24.8 \%$ fuel is saved during warmup on the FTP75, at an initial temperature of $-10^{\circ} \mathrm{C}$. By including warmup explicitly in the EMS, additional fuel is saved $(0.45 \%)$. The explicitly solved control map and costate dynamics show how the load on the ICE is increased, where the optimal balance is found between reduction of the warmup losses and usage of the battery system.

The purpose of our EMS is to minimize the fuel consumption, considering the ICE temperature of the HEV. There are however additional components that influence the fuel consumption, all with its own dynamics. E.g., the temperature of the battery influences the efficiency of the hybrid system, and will alter the results presented here, when explicitly taken into account in the EMS. However the insight on the costate behavior as presented here, paves the way for efficient real-time solutions on a vehicle. Extension of the optimal control based EMS for HEV with additional states, is part of future research.

\section{ACKNOWLEDGMENTS}

This work is supported by DAF Trucks N.V. and Agentschap NL under the HTAS Hybrid Innovations for Trucks project.

\section{REFERENCES}

1 Sciarretta A., Guzzella L. (2007) Control of hybrid electric vehicles, IEEE Control Systems Magazine 27, 2, 60-70.

2 Serrao L. (2011) Open issues in supervisory control of hybrid electric vehicles: a unified approach using optimal control methods. In Les Rencontres Scientifique d'IFP Energies nouvelles : les véhicules hybrides et électrifiés (RHEVE 2011) International Scientific Conference on hybrid and electric vehicles RHEVE 2011, 6-7 Dec., Ruel-Malmaison.

3 Willems F.P.T., Spronkmans S.J., Kessels J.T.B.A. (2011) Integrated powertrain control to meet low $\mathrm{CO}_{2}$ emissions for a hybrid distribution truck with SCR-de $\mathrm{NO}_{\mathrm{x}}$ system, In Proceedings of ASME 2011 Dynamic Systems \& Control Conference, 31 Oct.-2 Nov., Arlington, Virginia, pp. 1-6.

4 van Reeven V., Huisman R.G.M., Kessels J.T.B.A., Pham H.T., Hofman T. (2013) Integrating energy and thermal management of hybrid trucks, In Proceedings of the 12th International Conference Commercial Vehicles Trucks, Bus, Van, Trailer, 5-6 June, Celle, Germany, pp. 1-14, VDI.

5 Willems F., Kupper F., Rascanu G., Feru E. (2014) Integrated energy and emission management for Diesel engines with waste heat recovery using dynamic models, IFP Energies nouvelles International Conference: E-COSM'12 - IFAC Workshop on Engine and Powertrain Control, Simulation and Modeling, Oil \& Gas Science and Technology - Rev. IFP Energies nouvelles, doi: 10.2516/ogst/2013210.

6 Pham H.T., van den Bosch P.P.J., Kessels J.T.B.A., Huisman R.G.M. (2012) Integrated energy and thermal management for hybrid electric heavy duty trucks, In Proc. IEEE Vehicle Power and Propulsion Conference (VPPC), 09-12 Oct., Seoul, Korea.

7 Merz F., Sciarretta A., Dabadie J.-C., Serrao L. (2012) On the optimal thermal management of hybrid-electric vehicles with heat recovery systems, Oil \& Gas Science and Technology 67, 4, 601-612.

8 Kirk D.E. (1970) Optimal Control Theory, an introduction, Dover Publications.

9 Hartl R.F., Sethi S.P., Vickson R.G. (1995) A survey of the maximum principle for optimal control problems with state constraints, SIAM 37, 181-218.

10 Ambühl D., Sundström O., Sciarretta A., Guzzella L. (2010) Explicit optimal control policy and its practical application for hybrid electric powertrains, Control Engineering Practice 18, 12, 1429-1439. 
11 van Keulen T.A.C., Gillot J., de Jager A.G., Steinbuch M. (2014) Solution for state constrained optimal control problems applied to power split control for hybrid vehicles, Automatica 50, 187-192.

12 Kim N., Rousseau A., Lee D. (2011) A jump condition of PMPbased control for PHEVs, Journal of Power Sources 196, 10380-10386.

13 Willems F., Kupper F., Cloudt R., (2012) Integrated powertrain control for optimal $\mathrm{CO}_{2}-\mathrm{NO}_{\mathrm{x}}$ tradeoff in an Euro-VI Diesel engine with waste heat recovery system, In Proc. American Control Conference (ACC) 2012, 27-29 June, Montreal.

14 Lescot J., Sciarretta A., Chamaillard Y., Charlet A. (2010) On the integration for optimal energy management and thermal management of hybrid electric vehicles, In Proc. 2010 IEEE Vehicle Power and Propulsion Conference (VPPC), 1-3 Sept., Lille, pp. 1-6.

15 Guzzella L., Onder C. (2010) Introduction to Modeling and Control of Internal Combustion Engine Systems, Springer.

16 Heywood J.B. (1988) Internal Combustion Engines Fundamentals, McGraw-Hill.
17 van Berkel K., Klemm W., Hofman T., Vroemen B., Steinbuch M. (2013) Optimal control of a mechanical hybrid powertrain with cold start conditions, IEEE Trans Vehicular Technology 63, 4, 1555-1566.

18 Sandoval D., Heywood J.B. (2003) An improved friction model for spark-ignition engines, SAE paper 2003-01-0725.

19 Shayler P.J., Leong D.K.W., Murphy M. (2005) Contributions to engine friction during cold, low speed running and the dependence on oil viscosity, SAE paper 2005-01-1654.

20 Patton K.J., Nitschke R.G., Heywood J.B. (1989) Development and evaluation of a friction model for spark-ignition engines, SAE 890836.

21 Seeton C.J. (2006) Viscosity-temperature correlation for liquids, Tribology Letters 22, 67-78.

22 Holman J.P. (1992) Heat Transfer, McGraw.

23 Chachuat B. (2007) Nonlinear and dynamic optimization, Technical report, École Polytechnique Fédérale de Lausanne.

Manuscript submitted in March 2014

Manuscript accepted in September 2014

Published online in December 2014

Cite this article as: V. van Reeven, T. Hofman, F. Willems, R. Huisman and M. Steinbuch (2014). Optimal Control of Engine Warmup in Hybrid Vehicles, Oil Gas Sci. Technol 71, 14. 


\section{APPENDIX}

\section{A Modified FTP 75}

The FTP75 cycle is defined as a speed over time vector. For EMS comparison, a power over time vector is needed. Therefore a simple vehicle model is used to simulate the power demand over the drive cycle:

$$
P_{f t p}^{\prime}=v\left(0.5 \rho c_{w} A v^{2}+c_{r} m G+m \frac{d v}{d t}\right)
$$

with the parameters in Table 1 and $v$ and $t$ the prescribed FTP vectors. Heavy duty vehicles are often power limited, meaning that the cycle speed can not be tracked when the power limit is active. Here we simply limit the power cycle:

$$
P_{f t p}=\min \left(\max \left(P_{f t p}^{\prime}, \underline{P_{d}}\right), \overline{P_{d}}\right)
$$

which is justified for EMS comparison, but in effect shortens the covered distance, as the vehicle is not perfectly tracking the velocity profile. The resulting power demand is shown in Figure A1.

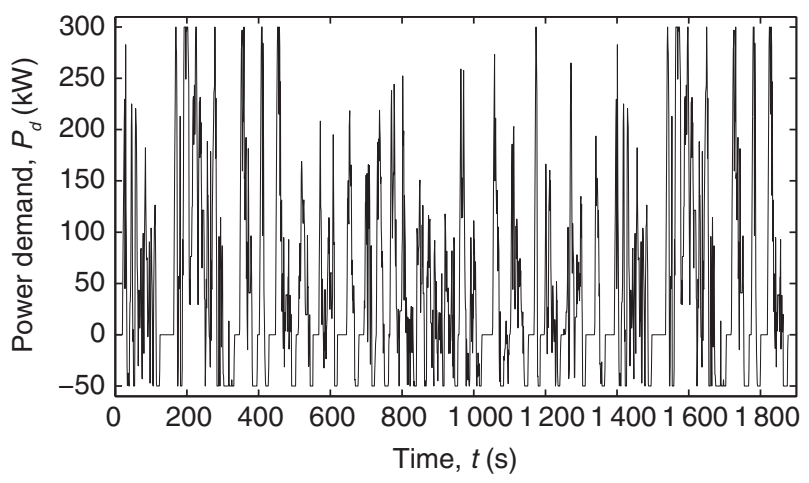

Figure A1

Modified FTP-75 as heavy-duty power cycle.

\section{B OCP Jump Conditions}

In the HEV the temperature of the ICE is not allowed to exceed a maximum limit, which constrains the temperature state. The time it touches the limit is not known beforehand, because it depends on the decision variables. If we assume that at time $t_{1}$ the maximum temperature is reached (Fig. A2), $d T / d t$ will be discontinuous. It is said that the state $T$ has a corner at junction time $t_{j}=t_{1}$, and results in a piecewise smooth state trajectory. For piecewise smooth trajectories, additional conditions can be derived, that describe the change in costate $\lambda$ and Hamiltonian $H$ at the corners. These are the so-called Weierstrass-Erdmann corner conditions and can be written as $[8,12]$ :

$$
-\left[\lambda_{j}^{-}-\lambda_{j}^{+}\right]^{T} \delta x_{j}+\left[H_{j}^{-}-H_{j}^{+}\right] \delta t_{j}=0
$$

where the superscript ${ }^{-}$and ${ }^{+}$denote the left-hand side and right-hand side limit values respectively, of junction $j$ at time $t_{j}$. Note the resemblance with the transversality condition in (36). If $x\left(t_{j}\right)$ and $t_{j}$ are unrelated, $\delta x_{j}$ and $\delta t_{j}$ can be independently 


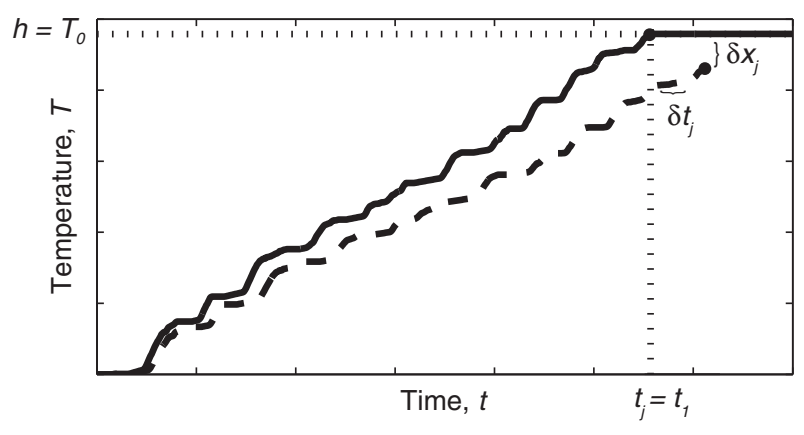

Figure A2

State $T$ having a corner at junction time $t_{1}$ when limit $T_{0}$ is touched.

varied, and thus their coefficients $\left(\lambda_{j}^{-}-\lambda_{j}^{+}\right.$and $\left.H_{j}^{-}-H_{j}^{+}\right)$must be zero. However, at $t_{j}$ we hit the state constraint and $x\left(t_{j}\right)$ and $t_{j}$ become related by state constraint $h$ :

$$
h(x(t), t) \leq 0
$$

As long as the state follows the constraint, the variation of $h$ must be zero:

$$
\delta h=\frac{\partial h}{\partial x} \delta x+\frac{\partial h}{\partial t} \delta t=0
$$

which gives the relation between $x\left(t_{j}\right)$ and $t_{j}$ along the constraint. Combining (A3) and (A5), results in the necessary conditions at the jump (38), (39), see [9]. 